\title{
O Grupo de Pesquisa Mobilidade e Novas Tecnologias de Interação do IFSP
}

\author{
Andre Constantino da Silva \\ Instituto Federal de São Paulo \\ Hortolândia \\ andre.constantino@ifsp.edu.br
}

\author{
Daniela Marques \\ Instituto Federal de São Paulo \\ Hortolândia \\ marquesdaniela@ifsp.edu.br
}

\author{
Gustavo Bartz Guedes \\ Instituto Federal de São Paulo \\ Hortolândia \\ gubartz@ifsp.edu.br
}

\begin{abstract}
RESUMO
Este artigo apresenta o grupo de pesquisa 'Mobilidade e Novas Tecnologias de Interação' do Instituto Federal de São Paulo câmpus Hortolândia que explora o potencial das diferentes modalidades de interação e mobilidade dos dispositivos computacionais, em especial, os móveis, em diferentes contextos de aplicação. Os principais projetos envolvem a investigação das modalidades, individualmente e conjuntamente, dos problemas ocasionados pela mudança de modalidade e na construção de software explorando os dispositivos disponíveis no laboratório considerando a usabilidade da aplicação.
\end{abstract}

\section{PALAVRAS-CHAVE}

Grupo de Pesquisa, Interação Humano-Computador, Multimodalidade, Dispositivos Móveis, Usabilidade.

\section{VISÃO GERAL}

O grupo de pesquisa "Mobilidade e Novas Tecnologias de Interação" do Instituto Federal de São Paulo câmpus Hortolândia nasceu da necessidade de estudar e produzir software que explore o potencial das diferentes modalidades de interação e da mobilidade fornecida pelos dispositivos móveis. Esta necessidade está intrinsicamente relacionada a um dos desafios da Interação Humano-Computador (IHC) apontado por [1]: "como garantir que os designs ofereçam usabilidade ao mesmo tempo que exploram o potencial e funcionalidade da nova tecnologia?".

Modalidade é o termo empregado para definir o modo como uma entrada do usuário e uma saída do sistema são expressas. $\mathrm{O}$ modo de comunicação se refere ao canal de comunicação usado por duas entidades diferentes que interagem [2]. Nigay e Courtaz [3] definem modalidade como um método de interação que um agente pode usar para alcançar uma meta. Segundo os autores, uma modalidade pode ser especificada em termos gerais como "usando fala" ou em termos mais específicos como "usando microfone".

Permission to reproduce or distribute, in whole or in part, material extracted from this work, verbatim, adapted or remixed, as well as the creation or production from the content of such work, is granted without fee for non-commercial use, provided that the original work is properly credited.

IHC 2019 - TRILHA FÓRUM DOS GRUPOS DE PESQUISA, Outubro 21-25, 2019, Vitória, Brasil. In Anais Estendidos do XVIII Simpósio Brasileiro sobre Fatores Humanos em Sistemas Computacionais. Porto Alegre: SBC.

(C) 2019 by the author(s), in accordance with the terms of the Creative Commons Attribution-NonCommercial 4.0 International Public License (CC BY-NC 4.0).
Ressalta-se que várias modalidades se tornaram foco de pesquisa nas últimas décadas, entre elas a fala (reconhecimento e sintetização), a escrita manuscrita (reconhecimento) e a interação com dedos por meio de toques e gestos. Assim, surgiram áreas de pesquisa dentro da Ciência da Computação que visam possibilitar a entrada e a saída de dados por uma modalidade, bem como explorar suas vantagens, como é o caso da Computação Baseada em Caneta e a interação por gestos.

Pensando na tecnologia dos computadores convencionais e na dos dispositivos móveis, acreditamos em uma complementação de uso dessas tecnologias. Dependendo da tarefa a ser realizada, utilizar um determinado hardware será mais rápido, eficiente e/ou oferecerá uma experiência mais gratificante aos usuários. É com esse pensamento que guiamos nossos trabalhos, no qual as diversas tecnologias (e as diferentes modalidades que essas tecnologias dão suporte) são utilizadas em prol das experiências dos usuários.

Na Seção 2 são apresentados os atuais membros do grupo de pesquisa. Na Seção 3, os objetos de pesquisa e, na Seção 4, a infraestrutura do grupo. Os principais projeto do grupo são apresentados na Seção 5 e, na Seção 6, finalizamos o artigo apresentando as oportunidades e desafios e nossos interesses de parceria.

\section{MEMBROS}

Criado e credenciado no diretório de pesquisa do CNPq em 2013, o grupo é formado atualmente por três professores-pesquisadores: o prof. Dr. André Constantino da Silva, líder do grupo, e os professores MSc. Daniela Marques e MSc. Gustavo Bartz Guedes.

Ao longo dos anos, contribuímos com a formação de mais de 20 discentes dispondo um espaço que permite aos interessados conhecer sobre modalidade e usabilidade de software, bem como Design Centrado no Usuário (DCU). Em 2019, nossa equipe é formada por um bolsista de iniciação científica na modalidade de Inovação Tecnológica com fomento oriundo da parceria IFSP e $\mathrm{CNPq}$, e diversos alunos de graduação que realizam o Trabalho de Conclusão de Curso orientados pelos pesquisadores.

\section{OBJETIVOS DO GRUPO}

Nossos objetivos relacionados ao contexto da IHC são:

- Explorar o potencial tecnológico relacionado ao suporte a diferentes modalidades e em diferentes contextos; 
- Estudar o impacto da mudança de modalidade na interação com o usuário;

- Desenvolver arcabouços, diretivas e aparatos tecnológicos para o desenvolvimento de aplicações envolvendo as diferentes modalidades;

- Auxiliar na formação de profissionais e pesquisadores nesta temática.

Dentre os diversos contextos de aplicação, desponta-se nosso interesse pela Educação, tentando responder a seguinte questão: "Qual a relação entre as modalidades existentes nos dispositivos computacionais com o seu emprego na Educação e como favorecer o ensino-aprendizagem empregando as novas modalidades de interação?". Assim, nossos objetivos relacionados ao contexto educacional são:

- Pesquisar o emprego de modalidades de interação, de forma individual e combinada, na educação presencial, híbrida e a distância;

- Desenvolver ferramentas educacionais que possibilitem o emprego das modalidades.

\section{INFRAESTRUTURA}

A Tabela 1 apresenta a diversidade de dispositivos disponíveis no laboratório. Essa variedade de dispositivos favorece o estudo de diferentes modalidades, individualmente e conjuntamente, apresentados a seguir.

Tabela 1. Dispositivos do Grupo de Pesquisa

\begin{tabular}{|l|l|l|}
\hline Dispositivo & Quant. & Modalidades de interação \\
\hline $\begin{array}{l}\text { Tablet Galaxy } \\
\text { Samsung Note 10 }\end{array}$ & 4 & $\begin{array}{l}\text { Tela sensível ao toque e à caneta; } \\
\text { microfone; câmera; caixa de som }\end{array}$ \\
\hline $\begin{array}{l}\text { Smartphone } \\
\text { Samsung Note 4 }\end{array}$ & 2 & $\begin{array}{l}\text { Tela sensível ao toque e à caneta; } \\
\text { microfone; câmera; caixa de som }\end{array}$ \\
\hline $\begin{array}{l}\text { Notebook Dell } \\
\text { Inspiron 13 }\end{array}$ & 2 & $\begin{array}{l}\text { Tela sensível ao toque è caneta; } \\
\text { teclado; mousepad; microfone; } \\
\text { câmera; caixa de som }\end{array}$ \\
\hline Notebook Dell & 1 & $\begin{array}{l}\text { Teclado; mousepad; microfone; } \\
\text { câmera; caixa de som }\end{array}$ \\
\hline Kinect v1 digital & 3 & \begin{tabular}{l} 
Sensor de movimentos corporais \\
\hline Kinect v2 ProInfo
\end{tabular} \\
\hline $\begin{array}{l}\text { Lousa } \\
\text { (modelo Projeção com sensibilidade à } \\
\text { FNDE/MEC [4]) }\end{array}$ & 1 & $\begin{array}{l}\text { caneta; teclado; mouse; } \\
\text { microfone; caixa de som }\end{array}$ \\
\hline LeapMotion & 2 & Sensor de movimento das mãos \\
\hline
\end{tabular}

\section{PRINCIPAIS PROJETOS}

A seguir, apresentamos os principais projetos desenvolvidos por meio de iniciações científicas, projetos de Trabalho de Conclusão de Curso e parcerias com outras instituições. Como embasamento teórico da IHC, empregamos o conceito de usabilidade [5], métodos de avaliação de usabilidade consagrados na literatura [1], e DCU [6].

\subsection{As diferentes modalidades no ensino técnico e superior}

Nesta temática, foram realizados dois projetos que visavam desenvolver uma sala de aula composta por equipamentos com diferentes modalidades (celulares, laptop, tablets e lousa interativa) explorando, de forma integrada, os recursos das modalidades de caneta, toque e gestos em atividades de ensinoaprendizado. Somos motivados pelo fato de o cérebro utilizar-se de estímulos dos diversos órgãos de sentido para um aprendizado mais eficaz. O projeto "Montagem de Laboratório de Investigação de Modalidade Multitoque em Contextos Educacionais", de 2013 a 2014, contou com auxílio institucional, e o projeto "Investigação da Interação Multimodal e de Mobilidade em Contextos Educacionais em Cursos Técnicos e Tecnológicos" com fomento do CNPq via Edital Universal (n 14/2014), de 2014 a 2018. Nesses projetos, um dos resultados é o EdiMM.

\subsection{EdiMM}

Nosso grupo, em parceria com pesquisadores do NIED/UNICAMP, vem desenvolvendo desde 2015 o Editor de Texto Multisemiótico Multimodal (EDIMM), uma aplicação para apoiar a elaboração de textos multisemióticos, textos que exploram as diferentes modalidades [7][8]. Os textos multisemióticos mais comuns (e antigos) são "os que agregam texto escrito e imagens e ainda hoje são fartamente utilizados em jornais, revistas e propagandas"[8]. Assim, os textos multisemióticos não são novidade, no entanto, acreditamos que "as tecnologias e, em especial, as tecnologias móveis, facilitam muito a produção de textos e hipertextos que incluem diferentes tipos de recursos expressivos, além da escrita" [8] e que o texto multisemiótico, produto da era digital/tecnológica, afeta não só as práticas sociais que envolvem a comunicação, mas também promove mudanças na própria linguagem [9].

O EdiMM também é uma ferramenta de interação multimodal pois seu projeto visa a utilização de diferentes modalidades de entrada (como teclado, voz, toque, mouse, caneta, gestos) para sua interação, explorando o contexto de multidispositivos. Atualmente o EdiMM possui interface responsiva (testado em computadores convencionais, laptops, smartphones, tablets e lousa digital) e, com trabalho em andamento para a inclusão de interação por (1) gestos e (2) por voz.

\subsection{Investigação das propriedades das modalidades de interação}

Neste tema, objetivamos investigar a relação entre as características de componentes da Interface Gráfica de Usuário (GUI - Graphical User Interface) quando usuários interagem com diferentes dispositivos de entrada em substituição ao mouse. Foram objetos de estudo em [10][11] a interação empregando dispositivo sensível ao movimento (WiiMote) e sensor de movimento (Kinect) ao interagir com uma aplicação especialmente desenvolvida com interface composta por diversos 
botões. Utilizamos, como procedimento metodológico, testes com usuário para mensuração da usabilidade.

\subsection{Investigação das mudanças de modalidade}

Com a evolução tecnológica, dispositivos com diferentes características acessam e renderizam as páginas e aplicações Web. Há também a necessidade de compatibilidade das aplicações em diferentes plataformas e, desta forma, aplicações são executadas em plataformas que não foram consideradas durante o seu desenvolvimento. Na interação com uma aplicação, cuja interface foi desenvolvida para uma modalidade, quando acessada por uma outra modalidade podem ocorrer problemas de interação oriundo da mudança de modalidade. Nosso grupo realiza estudos, como [10][11], para identificação desses problemas, estudando sua natureza e objetivando construir aparatos tecnológicos e diretivas que auxiliem os desenvolvedores que necessitam construir aplicações que serão usadas em diferentes modalidades de interação.

\subsection{Responsividade e modalidades}

Em [12] averiguamos que, a depender da tecnologia empregada no desenvolvimento, um objeto de aprendizagem (OA) desenvolvido para computadores desktop pode ser executado em um dispositivo móvel, mas os usuários podem se deparar com problemas de usabilidade. Para aumentar o número de usuários de um OA, é necessário que este seja acessível com boa usabilidade em diversas plataformas e com uma interface consistente entre elas. Em parceria com o Laboratório de Objetos de Aprendizagem da UFSCAR (LOA/UFSCar), estudamos como incluir a responsividade em jogos educacionais, como o jogos QuiMolecula, QuiForca e C.I.A. - Companhia de Investigação de Acasos. Na base metodológica para estes trabalhos utilizamos métodos de avaliação de usabilidade.

\subsection{Metodologias Ágeis e Design Centrado no Usuário}

Outra preocupação do grupo de pesquisa é o processo de desenvolvimento de software e a integração entre a IHC e a Engenharia de Software. Dentre nossos trabalhos realizados, destacamos o projeto FilantroTeam, que integra Scrum e DCU para o desenvolvimento o aplicativo Ant [13], uma aplicação para dispositivos móveis com propósito de aproximar voluntários e instituições sem fins lucrativos. Percebe-se, por meio dos projetos, caraterísticas comuns entre as metodologias ágeis e o DCU, como ambos serem iterativos, incrementais e envolver os usuários desde o início do processo de desenvolvimento, o que possibilita uma integração entre as áreas.

\section{DESAFIOS, OPORTUNIDADES E INTERESSES DE PARCERIA}

A partir de nossas experiências com os projetos citados, identificamos os seguintes desafios de pesquisa:

- O potencial das modalidades e a complementação entre elas: Como explorar o potencial de uma modalidade de interação?
Como as modalidades podem se complementar? Como o contexto pode interferir na escolha do usuário em relação as modalidades? Como suportar atividades que podem se iniciar em um dispositivo (que possui um conjunto de modalidade) e migrar para outro dispositivo (com outro conjunto de modalidade)?

- Multimodalidade, usabilidade e acessibilidade: Como a interação multimodal impacta na usabilidade e na acessibilidade de uma aplicação? Quais são os benefícios e limitações de softwares multimodais em relação aos usuários com deficiência?

- Desenvolvimento de software diferentes modalidades: Como auxiliar os desenvolvedores no contexto de desenvolvimento de aplicações para diferentes modalidades? Como desenvolver software no contexto de multidispositivos e multimodalidade? E como facilitar o desenvolvimento de software neste contexto?

Esses desafios, apresentam-se como oportunidades de pesquisa tecnológica visto o impacto dos dispositivos computacionais na sociedade. Desta forma, procuramos parcerias com instituições públicas e privadas, bem como com outros grupos de pesquisa, visando aplicar nossos conhecimentos em projetos existentes ou em desenvolvimento de projetos com a temática apresentada.

\section{REFERÊNCIAS}

[1] Y. Rogers, H. Sharp, and J. Preece. 2013. Design da Interação: além da interação homem-computador (3. ed.). Bookman, Porto Alegre, RS.

[2] J. Alty and C. McCartney. 1991. Design of a Multi-Media Presentation System For A Process Control Environment. In Proceedings Eurographics Multimedia Workshop, Session 8: Systems. Springer-Verlag, Berlin.

[3] L. Nigay and J. Courtaz. 1995. A Generic Platform for Addressing the Multimodal Challenge. In Proceedings of 13 SIGCHI Conference on Human Factors in Computing System (CHI'95). ACM Press/Addison-Wesley Publishing Co., New York, NY, 98-105. DOI: https://doi.org/10.1145/223904.223917.

[4] MEC. FNDE - MEC - Ministério da Educação. Computador Interativo e Lousa Digital (Projetor Proinfo). Disponível em http://www.fnde.gov.br/portaldeco mpras/index.php/produtos/computador-interatvo-projetor.

[5] J. Nielsen. 1993. Usability Engineering. Academic Press, Boston, MA.

[6] ISO/IEC. 9241-210:2010 Ergonomics of human-system interaction - Part 210: Human-centred design for interactive systems (E), 2010.

[7] F. L. Arantes, F.M.P. Freire, J. Breuer, A.C. da Silva, R.C.A. Oliveira, and L.E.L. Vascon. 2017. Towards a Multisemiotic and Multimodal Editor. Journal of Computer Science and Technology, 17, 02 (Oct. 2017), 100-109. DOI: https://doi.org/10.24215/16666038.17.e14 .

[8] F.M.P. Freire, F.L. Arantes, A.C. da Silva, and L.E.L. Vascon. 2015. Estudo de viabilidade de um Editor Multimodal: o que pensam os alunos?. In Anais do XX Congreso Internacional de Informática Educativa (TISE 2015). Universidad de Chile, Santiago, volumen 11, 109-119.

[9] D.B. Braga and I. Ricarte. 2005. Letramento e Tecnologia. UNICAMP Cefiel \& MEC, Campinas, SP.

[10] A.C. da Silva, G.D.R. Farias, A.S.F. Gomes, and F.E.R. Silva. 2015. Identifying Interaction Problems on Web Applications Due to the Change of Input Modality: a study about Kinect, Google Maps and Google Street View. In Proceedings of 14th International Conference WWW/Internet (WWW/Internet 2015). IADIS Press, 192-196.

[11] A.C. da Silva, L.O.A. Nascimento, and V.S.S. Silva. 2017. User Interaction Using Motion Sensitive Devices: a study about button's dimension with Kinect and WiiMote. In Proceedings IADIS International Conference Applied Computing 2017 (AC 2017). IADIS Press, 314-317.

[12] M.D.O. da Cruz and A.C. da Silva. 2014. Investigação da Portabilidade e Usabilidade de Conteúdos Educacionais Brasileiros em Celulares da Plataforma Android. In Anais do 5 Congresso de Iniciação Científica e Tecnológica do IFSP. IFSP, São Paulo, SP.

[13] A.C. da Silva, D.R.S. Silva, D. Marques, G.B. Guedes, F.O.V. Pinto, and V.S Silva. 2017. Associando Scrum e Projeto Centrado no Usuário: um estudo de caso no desenvolvimento da aplicação móvel Ants. In Atas das Conferências Ibero Americana WWW/Internet e Computação Aplicada 2017 (CIACA 2017). IADIS Press, 263-266. 